\title{
Pathogen germs response to low-dose radiation - medical approach
}

\author{
A. Pointa ${ }^{1 *}$, R. Focea ${ }^{2}$ and D. Creanga ${ }^{2}$ \\ ${ }^{1}$ University of Medicine and Pharmacy "Gr. T. Popa" \\ Faculty of Pharmacy - Iasi, Romania \\ 2 "Al. I. Cuza" Univesity, Faculty of Physics - Iasi, Romania
}

\begin{abstract}
The side effects of radiation therapy in the case of microbial loading of irradiated organs was considered as phenomenological basis of the experiment carried out on Staphylococcus aureus (ATCC germ) exposed to low X-ray doses. The inoculum was prepared in a liquid culture medium with standard composition, the volumes of $3 \mathrm{ml}$ identical samples (in sterile glass tubes) being irradiated in hospital conditions. Five experimental variants were developed corresponding to irradiation time durations between 25 and 100 minutes. The spectro-colorimetric assay was accomplished at $560 \mathrm{~nm}$ and $420 \mathrm{~nm}$, the resulting average values (for three repetitions) being analyzed from the viewpoint of cell density in the irradiated variants compared to control ones. The resistance to antibiotics of the irradiated bacteria was tested on agarized cultures against five antibiotic molecules (ampicillin, cloramphenicol, tetracycline, tobramicin and ofloxacin) by assessing the diameter of inhibition growth areas in each case. The increase of the inhibition area diameter with up to $15 \%$ (in the case of tetracycline) was noticed for the lowest irradiation time for all five antibiotics, which is suggesting a weakening of the bacteria resistance to the pharmaceutical agents following the X-ray treatment. This was concordant with the results of the spectro-colorimetric assay of the cell density within the directly irradiated bacteria cultures. The main issue of this study is concerning
\end{abstract}

*E-mail: ramona_focea@yahoo.com

This is an Open Access article distributed under the terms of the Creative Commons Attribution License 2.0, which permits unrestricted use, distribution, and reproduction in any medium, provided the original work is properly cited. 
the optimization of the radiotherapy protocol in patients with potential microbial loading.

\section{Introduction}

Cancer and its treatments lead to profound suppression of innate and acquired immune function. In this population, bacterial infections are common and may rapidly lead to overwhelming sepsis and death. Furthermore, infections caused by viral and fungal pathogens should be considered in patients who have specific immune defects. As cancer therapies have become more aggressive, the risk for infection has increased and many patients require intensive care support. Despite improvements in long-term survival, infections remain a common complication of cancer therapy and accounts for the majority of chemotherapy-associated deaths.

Staphylococcus aureus is a versatile organism provided with several virulent characteristics and resistance mechanisms. It is also the widely spread cause of various infectious diseases in humans. S. aureus often causes life-threatening deep seated infections like bacteremia, endocarditis and pneumonia. While traditionally confined mostly to the hospital setting, methicillin-resistant $S$. aureus (MRSA) is now rapidly becoming rampant in the human community. Community-acquired MRSA is particularly significant because of its potential for unchecked spread within households and its propensity for causing serious skin and pulmonary infections.

The possible side effects of radiation therapy in the case of microbial loading of irradiated organs was considered as phenomenological basis of the experiment carried out on Staphylococcus aureus (ATCC 25923 germ) exposed to X-ray irradiation. The purpose of this study was to highlight the influence of radiation doses comparable to those used in radiotherapy (relatively low doses compared to those required for sterilization of microorganisms) on $S$. aureus growth as well as on its resistance to various types of antibiotics. Previous experiments allowed the assessing of the (electro) magnetic fields effects on various bacteria resistance to several antibiotics $[1,2]$, the general evidenced tendency being that of bacteria resistance diminution to some antibiotics. Cancer patients, because of their immunocompromised state and antibiotic treatment, may be susceptible to opportunistic infections and to organisms that usually cause minimal infection. On the other hand, there is much evidence that the inappropriate use of antibiotics may be harmful by encouraging the colonisation of resistant organisms [3]. 


\section{Experimental}

For $\boldsymbol{X}$-ray exposure we used a specialized X-ray system for radiation therapy of skin lesions. The system provides X-ray emission of low energy, characterized by $70 \mathrm{kV}$ and $10 \mathrm{~mA}$, with an $0.75 \mathrm{~mm} \mathrm{Al}$ filtration and $\mathrm{HVL}$ of $1.3 \mathrm{~mm} \mathrm{Al}$ equivalent. The dose rate was of $227 \mathrm{cGy} \mathrm{min}^{-1}$, measured at a source - to- skin distance of $25 \mathrm{~cm}$.

Microbiological material was represented by bacterial strain samples from standard collections of Staphylococcus aureus (ATCC 25923 germ). The inoculum was prepared in liquid culture medium with standard composition, $3 \mathrm{ml}$ equal volumes (in sterile glass tubes) being irradiated in hospital conditions. Five experimental variants were developed corresponding to irradiation times between 25 and 100 minutes (with absorbed dose values of 31 Gy, 62 Gy, 87 Gy, 107 Gy and 128 Gy).

The spectro-colorimetric assay was accomplished at $560 \mathrm{~nm}$ and $420 \mathrm{~nm}$. The resulting average values (for three repetitions of the whole experiment) have been analyzed from the viewpoint of cell density in the irradiated variants compared to the control samples. The Shimadzu Spectrophotometer UV type 1700 Pharmaspec with quartz cells was used for the turbidimetric measurements.

For measuring antibiotic resistance, the Kirby-Bauer [4] method was used. The resistance to antibiotics of the irradiated bacteria was tested on agarized cultures against five antibiotic molecules (ampicillin (A), chloramphenicol $(\mathrm{C})$, tetracycline $(\mathrm{T})$, tobramycin (TOB) and ofloxacin $(\mathrm{OF})$ ) by assessing the diameter of inhibition growth areas in each case.

\section{Results and discussion}

According to the average values reported in Fig. 1, the growth inhibition discs of $S$. aureus in the presence of tobramycin were slightly increased for 24 minutes exposure to X-rays while for longer time duration of radiation action no significant changes compared to the control samples could be noticed so that considering the average standard deviation we might say that the resistance to tobramycin did not changed after irradiation.

In the case of two other antibiotic drugs: ofloxacin and ampicillin the longest irradiation time appeared to have some significant effect on the growth of $S$. aureus bacteria (Figs. 2-3) - consisting in the diminution of the inhibition growth area but generally bacteria resistance to ofloxacin and ampicillin may be considered as not changed following the radiation action. 


\section{Staphylococcus aureus resistance to}

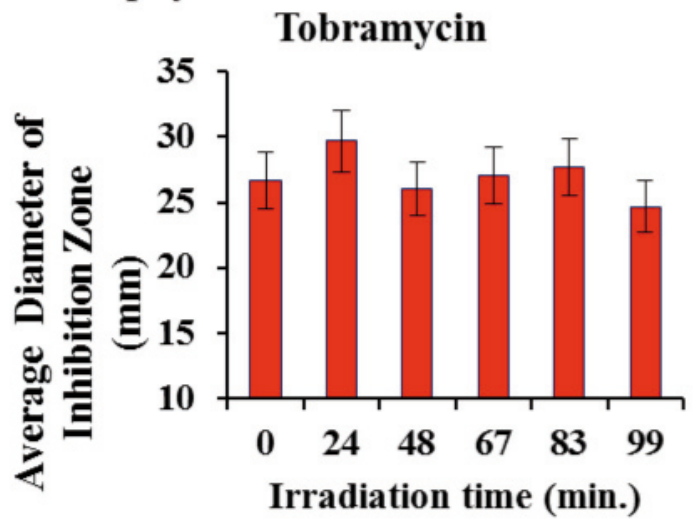

Fig. 1: The resistance to tobramycin of irradiated S. aureus cells.

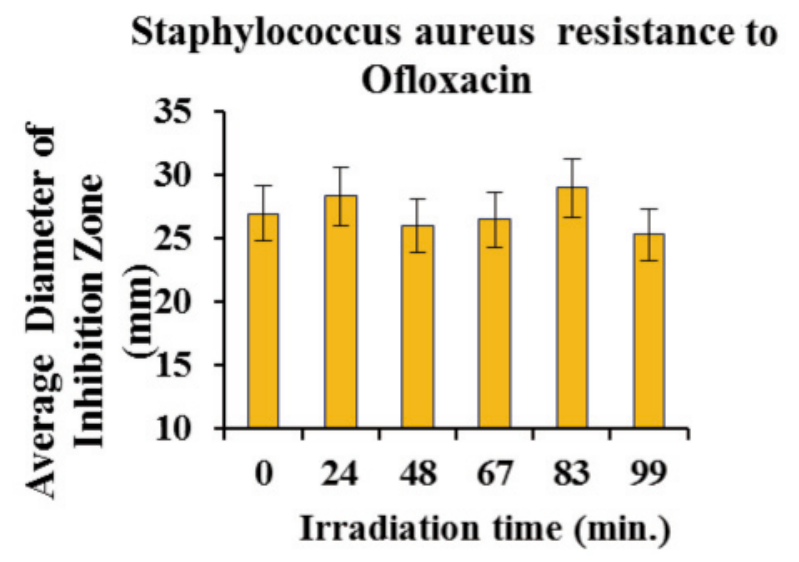

Fig. 2: The resistance to ofloxacin of irradiated $S$. aureus cells. 


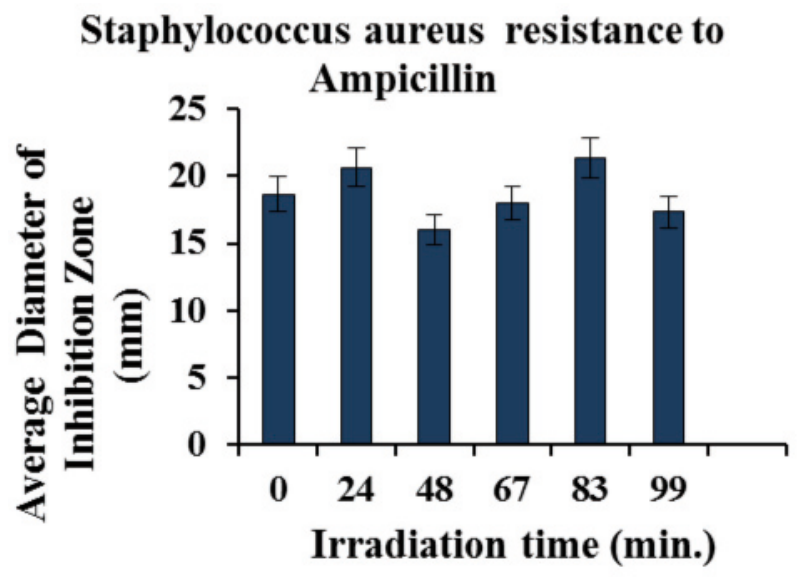

Fig. 3: The resistance to ampicillin of irradiated S. aureus cells.

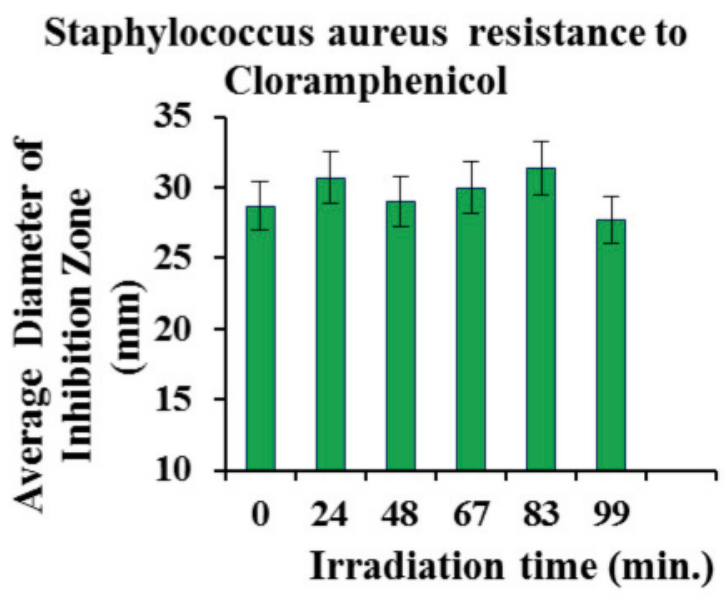

Fig. 4: The resistance to chloramphenicol of irradiated $S$. aureus cells.

In the case of chloramphenicol and tetracycline (Figs. 4-5) the changes revealed in the microbial samples exposed to X-ray action are similar to the standard deviation order of magnitude so the conclusion is that no important modifications were induced at the levels of the cellular targets for the two antibiotics.

Cell density variations (Fig. 6) in the directly irradiated tubes exhibit remarkable diminution around the X-ray exposure time of 50 minutes where the damages caused by the absorption of the radiation energy probably affected seriously the cell viability and consequently their proliferative ac- 


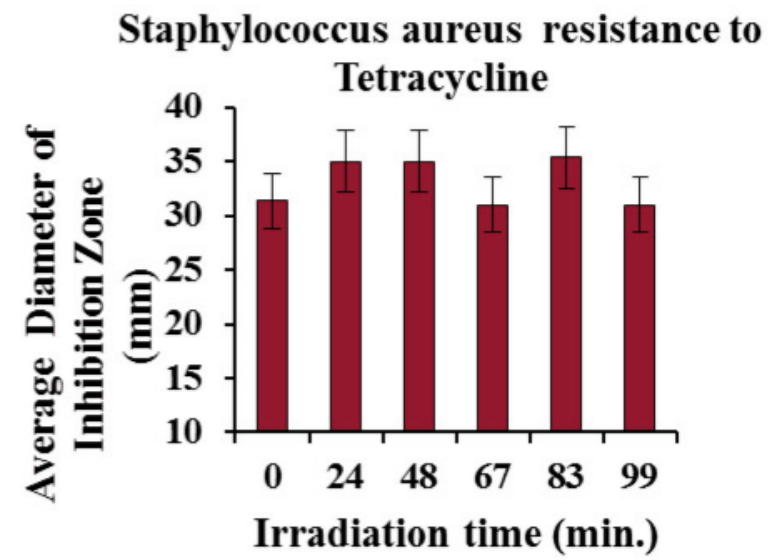

Fig. 5: The resistance to tetracycline of irradiated S. aureus cells.

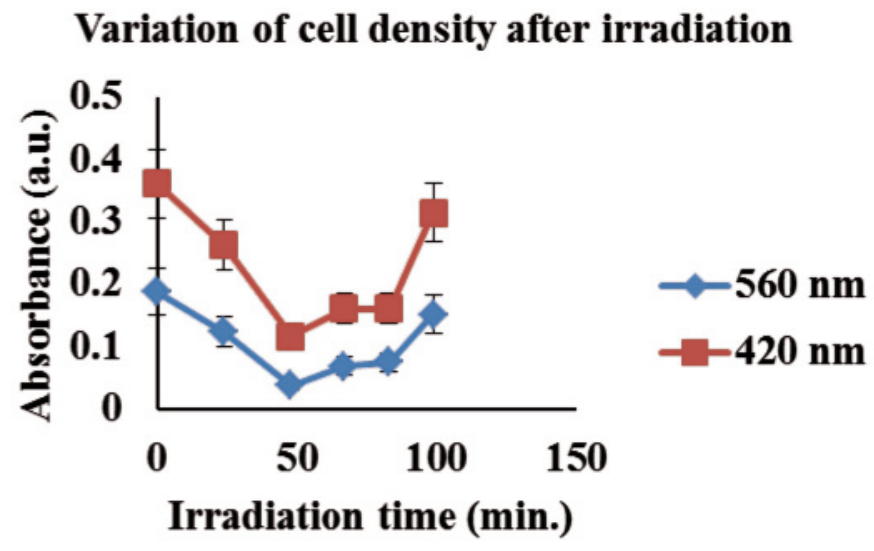

Fig. 6: The cell density response to the low doses of X-radiation administration.

tivity. The cell density was reduced more than twice- as resulted from the data provided by spectral measurements at $560 \mathrm{~nm}$ and at $420 \mathrm{~nm}$. Nevertheless, no visible correlation with the diameters of the inhibition growth zones could be found.

\section{Conclusions}

The $S$. aureus response to low X-ray doses was evidenced mainly at the level of the cell density values in the liquid culture medium that was directly exposed to X-rays. The resistance to various antibiotic drugs seems not 
to be a good indicator of the radiation effect since no significant changes were noticed in the growth inhibition area - as tested on agarized cultures. From the medical viewpoint, however, the resistance to antibiotics is crucial when the patient health is concerned, so, the main conclusion of this work is that no significant influence of the patient's exposure to low radiation doses (usual in the radiation therapy) is expected for the efficacy of an additional antibiotic treatment at the level of the microbial loaded organs.

\section{Acknowledgements}

This work was partially supported by the European Social Fund in Romania, under the responsibility of the Managing Authority for the Sectorial Operational Programme for Human Resources Development 2007-2013 [grant POSDRU/88/1.5/S/47646].

\section{References}

[1] CREANGA D. E., et al., J. Magn. Magn. Mater. 272-276(III) (2004) 2442 .

[2] POIATA A., et al., Electromagn. Biol. Med. 22(2-3) (2003) 171.

[3] LIVERMORE D., Intensive Care Med 26 (Suppl. 1) (2000) S14-S21.

[4] BAUER R. W., et al., American J. Clinical Pathology 45 (1966) 493-496. 